\title{
A Comparative Study on Consumers' Preference of Processed Spices in Bangalore and Chennai City
}

\author{
V. M. Indumathi ${ }^{1 *}$, M. Malarkodi ${ }^{2}$ and S. Jesupriya Poornakala ${ }^{1}$ \\ ${ }^{1}$ KVK, Vamban, Pudukkottai, India \\ ${ }^{2}$ Directorate of Agribusiness Development, TNAU, Coimbatore, India \\ *Corresponding author
}

\section{A B S T R A C T}

Keywords

Processed Spice,

Consumer

Preference,

Consumers, Brands

Article Info

Accepted:

17 October 2020

Available Online:

10 November 2020
During the last few years, new opportunities in the processed food sector like nutritive foods, spices, ready to cook food items like chapattis, vegetable dishes, pickles, snack foods etc., have been gaining wider acceptance. Consumers have been looking for more variety and new products. Simple random sampling procedure was followed and ultimately 200 consumers were selected. Good quality, product variety, brand image and attractive package are essential to attract customers. Brands of processed spices consumed in two cities were significantly different.

\section{Introduction}

Globalization had an impact on the homogenization of consumer behaviour with respect to food and grocery in the last decade or so and the need for convenience, good health, choice and more value of money has been the emerging trends in food sector. Clear shifts towards ready to cook and ready to eat products, which can help consumer save time and effort can be seen in processed foods segment. Consumers have been looking for more variety and new products. This has been reflected in the fact that majority of spending on food had been in supermarkets and hypermarkets which offered thousands of products on shelves for customers to choose. During the last few years, new opportunities in the processed food sector like nutritive foods, spices, ready to cook food items like chapattis, vegetable dishes, pickles, snack foods etc., have been gaining wider acceptance.

According to Kathuria et al., (2013) the consumers actually aware of the brand they buy and found the source of awareness. Conventionally, it is thought that consumers tend to buy familiar brands out of habit or because of loyalty, and they are likely willing 
to pay more for a branded product than for a non-branded generic product. Familiar or popular brands can induce the placebo effect in a consumer and elevate a consumer's satisfaction derived through heightened taste perceptions of food products (Spinelli et al., 2015). Xie et al., (2014) examined that consumers expected more safety and healthier to organic process food in China. These kinds of products were purchased by the higher level of education and economically sound customers. Wang (2013) describe that visual packaging is more important consideration to influence the consumers to perceive value, quality of the commodity and brand preference effect on the value of the food product. Richie et al., (2017) shown that most of the consumers didn't select the national brand instead they used private brands.

\section{Materials and Methods}

Simple random sampling technique was used to select area, supermarkets and sample respondents. In Bangalore and Chennai ten consumers per super market with self service facilities were selected at random. In each store among the consumers who purchased from the store on the days of visit, ten consumers were selected at random. Since the study pertains to use of spices, only women consumers were selected as respondents. Thus, a simple random sampling procedure was followed and ultimately 200 consumers were selected. For collection of primary data a well-structured interview schedule was prepared based on the objectives of the study. Simple percentages and averages were worked out to study the general characteristics of the sample consumers like age, educational status, occupation, family size and type, purchase of processed spice products etc.

Different processed spice products were available in the market. The consumers used varying quantities of different spice products based on their need. An attempt was made to test whether the quantity of different spice products consumed by respondents at Chennai and Bangalore were the same. For this purpose Wilcoxon - Mann - Whitney test (Kothari, 1998) was done. This test is used to determine whether two independent samples have been drawn from the same population. To perform this test, we first rank the data jointly, taking them as belonging to a single sample in either an increasing or a decreasing order of magnitude. Usually low to high ranking process which means assign rank 1 to an item with lowest value, rank 2 to the next highest item and so on. In case of ties the average of the rank was given but in this case there was no tie in between the rank. Then we have to find the sum of the ranks assigned to the values of the first sample and also the sum of the ranks assigned to the values of which is a measurement of the difference between the ranked observations of the two samples as under;

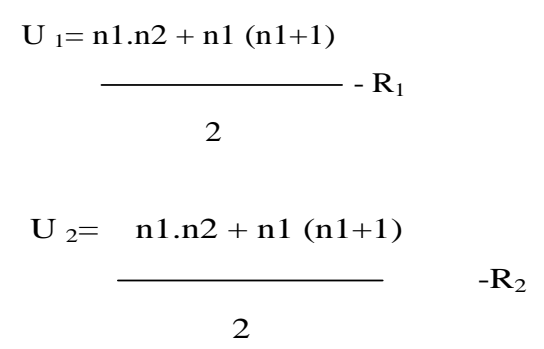

Where $\mathrm{n} 1$ and $\mathrm{n} 2$ are the sample sizes

$\mathrm{R}_{1}$ is sum of ranks assigned to the values of the first sample.

$\mathrm{R}_{2}$ is sum of ranks assigned to the values of the second sample.

The null hypothesis is that two samples come from identical populations, If this hypothesis is true, it seems reasonable to suppose that the means of the ranks assigned to the values of the two samples should be more or less the 
same. Under the alternative hypothesis, the means of the two populations are not equal and if this is so, then most of smaller ranks will go to the values of one sample while most of the highest ranks will go to those of the other sample. If the null hypothesis that the $n 1+n 2$ observations came from identical population is true, the said ' $u$ ' statistic has a sampling distribution with

Mean $=\mu_{\mathrm{u}}=\mathrm{n} 1 . \mathrm{n} 2$

2

and Standard deviation (or the standard error)

$$
\sigma_{\mathrm{u}}=\mathrm{n} 1 \mathrm{n} 2(\mathrm{n} 1+\mathrm{n} 2+1)
$$

12

The sampling distribution of $U$ can be approximated closely with normal distribution and the limits of the acceptance region can be determined in the usual way at a given level of significance. In this study, this analysis is used to test whether there is a difference in the quality of processed spice products consumed between Chennai and Bangalore.

The hypothesis is $h_{0}$ : There is no difference between the consumption of processed spice products in two cities.

$\mathrm{h}_{1}$ : There is a significant difference between the consumption of processed spice products in two cities.

\section{Results and Discussion}

\section{General characteristics of the sample respondents}

The general characteristics of the sample respondents included the age, education status, occupation, family size and type, number of educated persons, working members of the family and annual income of the family. The data on the above aspects were analysed to understand their preference for processed spices for culinary purposes.

\section{Age of the respondents}

The details regarding the age of the sample respondents are given in Table 1 . It could be observed that majority (77 per cent) of the sample respondents belonged to the age group of 36 to 55 years. Both the cities recorded higher share of respondents in the above age group only. Bangalore and Chennai had the lowest proportion of sample respondents in the age group of above 55 years. It could be concluded that majority of the respondents in both the cities were middle aged.

\section{Educational status of the respondents}

The educational background has profound influence on the preference of a particular brand. Therefore, the respondents were categorized into three groups based on their education and the results are presented in Table 2.

It could be inferred from the table that majority of the respondents had graduate education (53 per cent) followed by post graduation (33 per cent). In Chennai most of the respondents (56 per cent) were graduates. In Bangalore, the proportion of postgraduates was higher (38 per cent) when compared to Chennai. It could be, inferred that all the respondents were educated and would have knowledge on processed foods.

\section{Occupation of the respondents}

The occupational status was analysed as it indicates the time available to respondents for cooking, which in turn influences the purchase of processed food products. The details regarding occupational status are furnished in Table 3. 
It could be seen from the above table that 57 per cent of the sample respondents were employed. Bangalore had highest percentage of working population (67 per cent) when compared to Chennai. In contrast, the population of housewives was high in Chennai (54 per cent). Since most of the respondents were employed, the usage of processed foods was expected to be higher.

\section{Family type}

The type of the family would influence the consumption, usage, and purchase of processed spice products. Hence, the family type was divided into two categories namely joint and nuclear. The details about the family type are presented in Table 4.

It could be observed from the table that 91 per cent of the respondents belonged to the category of nuclear family. The percentage of nuclear family in Bangalore and Chennai was 93.00 and 89.00 per cent, respectively. It could be concluded that majority of the respondents in both the cities belonged to nuclear family category, hence decision making would be easier and also the usage of processed food may be more.

\section{Number of educated members in the family}

The educated members in a family would influence the knowledge about new products and usage of premium products. The details regarding number of educated members in the households of sample respondents are presented in Table 5.

Majority of the family (52 per cent) had three educated members in a family. In Bangalore majority of the families (55 per cent) had three educated members but the Chennai city recorded only 48 per cent in this category. About 42 per cent of Chennai respondents' family had four educated members per family as against 31per cent in Bangalore in the same group. It could be inferred that majority of the families in both the cities had three educated members per family.

\section{Working persons in the family}

The total number of working persons in a family decides the income of the particular family and it directly influences the expenditure pattern of a family. The maximum number of working persons in a family was three and the details are presented in Table 6.

It could be found that most of the families (48 per cent) in the study area had two working persons per family followed by three members in a family. About 53 per cent of Bangalore families had two working members whereas in Chennai it was only 43 per cent with two members. The families having only one person working were high (23 per cent) in Chennai when compared to Bangalore (8 per cent). It could be concluded that majority of the families had two or three working persons, hence the usage of processed food products is likely to be more.

\section{Annual income of the family}

The details about the annual income of the family of the respondents are important as it reflects the purchasing power of the consumers. It also influences the total expenses for food and its related products. The spending habit differs from one family to another and also from place to place. The data on the income was collected, analysed and the results are presented in Table 7.

It could be inferred from the table that 33 per cent of the respondents were having an annual family income of Rs. 2.1 to 2.5 lakhs followed by nearly 30 per cent of the families 
earning Rs. 2.6 to 3.0 lakhs. About 39 per cent of the sample respondents in Bangalore had a family increase of Rs.2.6 to 3.0 lakhs when compared to 32 per cent in Chennai. Around 23 per cent of the total respondents in the both the cities had the annual family income of Rs 3.1 to 3.5 lakhs. It could be concluded that majority of the households were in high income group, hence they would be interested in processed foods.

Table.1 Age of the respondents (Number of respondents)

\begin{tabular}{|l|l|c|c|c|}
\hline S.No & Age & Bangalore & Chennai & Overall \\
\hline $\mathbf{1 .}$ & $\mathbf{2 5 - 3 5}$ & 17 & 11 & $\mathbf{2 8}$ \\
& & $(17.00)$ & $(11.00)$ & $(\mathbf{1 4 . 0 0})$ \\
\hline $\mathbf{2 .}$ & $\mathbf{3 6 - 4 5}$ & 38 & 43 & $\mathbf{8 1}$ \\
\hline $\mathbf{3 .}$ & $\mathbf{4 6 - 5 5}$ & $(38.00)$ & $(43.00)$ & $\mathbf{( 4 0 . 5 0 )}$ \\
\hline $\mathbf{4 .}$ & $\mathbf{5 6 - 6 5}$ & 33 & 39 & $\mathbf{7 2}$ \\
& & $(33.00)$ & $(39.00)$ & $\mathbf{( 3 6 . 0 0 )}$ \\
\hline & Total & 12 & 7 & $\mathbf{1 9}$ \\
& & $(12.00)$ & $(7.00)$ & $\mathbf{( 9 . 5 0 )}$ \\
\hline
\end{tabular}

(Figures in parentheses indicate percentage to total)

Table.2 Educational Status of the Respondents (Number of respondents)

\begin{tabular}{|l|l|c|c|c|}
\hline S.No & \multicolumn{1}{|c|}{ Education } & Bangalore & Chennai & Overall \\
\hline $\mathbf{1 .}$ & PreUniversity Class & 13 & 17 & $\mathbf{3 0}$ \\
& & $(13.00)$ & $(17.00)$ & $(\mathbf{1 5 . 0 0})$ \\
\hline 2. & Graduate & 49 & 56 & $\mathbf{1 0 5}$ \\
& & $(49.00)$ & $(56.00)$ & $\mathbf{( 5 2 . 5 0 )}$ \\
\hline $\mathbf{3 .}$ & Post graduate & 38 & 27 & $\mathbf{6 5}$ \\
& & $(38.00)$ & $(27.00)$ & $\mathbf{( 3 2 . 5 0 )}$ \\
\hline & Total & $\mathbf{1 0 0}$ & $\mathbf{1 0 0 . 0 0}$ & $\mathbf{2 0 0}$ \\
& & $\mathbf{( 1 0 0 . 0 0 )}$ & $\mathbf{( 1 0 0 . 0 0})$ & $\mathbf{( 1 0 0 . 0 0 )}$ \\
\hline
\end{tabular}

(Figures in parentheses indicate percentage to total)

Table.3 Occupation of the respondents

\begin{tabular}{|l|l|r|r|r|}
\hline S.No & Occupation & Bangalore & Chennai & \multicolumn{1}{c|}{ Overall } \\
\hline $\mathbf{1 .}$ & Employed & 67 & 46 & $\mathbf{1 1 3}$ \\
\hline $\mathbf{2 .}$ & Housewife & $(67.00)$ & $(46.00)$ & $\mathbf{( 5 6 . 5 0 )}$ \\
\hline & Total & 33 & 54 & $\mathbf{8 7}$ \\
& & $(33.00)$ & $(54.00)$ & $\mathbf{( 4 3 . 5 0 )}$ \\
\hline
\end{tabular}

(Figures in parentheses indicate percentage to total) 
Table.4 Family type (Number of respondents)

\begin{tabular}{|l|l|c|c|c|}
\hline S.No & Family type & Bangalore & Chennai & Overall \\
\hline $\mathbf{1 .}$ & Joint & 7 & 11 & $\mathbf{1 8}$ \\
& & $(7.00)$ & $(11.00)$ & $(\mathbf{9 . 0 0})$ \\
\hline $\mathbf{2 .}$ & Nuclear & 93 & 89 & $\mathbf{1 8 2}$ \\
& & $(93.00)$ & $(89.00)$ & $\mathbf{( 9 1 . 0 0 )}$ \\
\hline & Total & $\mathbf{1 0 0}$ & $\mathbf{1 0 0}$ & $\mathbf{2 0 0}$ \\
& & $\mathbf{( 1 0 0 . 0 0 )}$ & $\mathbf{( 1 0 0 . 0 0 )}$ & $\mathbf{( 1 0 0 . 0 0 )}$ \\
\hline
\end{tabular}

(Figures in parentheses indicate percentage to total)

Table.5 Number of educated members in the family (Number of respondents)

\begin{tabular}{|c|c|c|c|c|}
\hline S.No & $\begin{array}{l}\text { No. of educated } \\
\text { members }\end{array}$ & Bangalore & Chennai & Overall \\
\hline 1. & Two & $\begin{array}{c}14 \\
(14.00)\end{array}$ & $\begin{array}{c}10 \\
(10.00)\end{array}$ & $\begin{array}{c}24 \\
(12.00)\end{array}$ \\
\hline 2. & Three & $\begin{array}{c}55 \\
(55.00)\end{array}$ & $\begin{array}{c}48 \\
(48.00)\end{array}$ & $\begin{array}{c}103 \\
(51.50)\end{array}$ \\
\hline 3. & Four & $\begin{array}{c}31 \\
(31.00)\end{array}$ & $\begin{array}{c}42 \\
(42.00)\end{array}$ & $\begin{array}{c}73 \\
(36.50)\end{array}$ \\
\hline & Total & $\begin{array}{c}100 \\
(100.00)\end{array}$ & $\begin{array}{c}100 \\
(100.00)\end{array}$ & $\begin{array}{c}200 \\
(100.00)\end{array}$ \\
\hline
\end{tabular}

(Figures in parentheses indicate percentage to total)

Table.6 Working persons in the family (Number of respondents)

\begin{tabular}{|c|c|c|c|c|}
\hline S.No & $\begin{array}{l}\text { No. of working } \\
\text { persons }\end{array}$ & Bangalore & Chennai & Overall \\
\hline 1. & Only one & $\begin{array}{c}8 \\
(8.00)\end{array}$ & $\begin{array}{c}26 \\
(26.00)\end{array}$ & $\begin{array}{c}34 \\
(17.00)\end{array}$ \\
\hline 2. & Two & $\begin{array}{c}53 \\
(53.00)\end{array}$ & $\begin{array}{c}43 \\
(43.00)\end{array}$ & $\begin{array}{c}96 \\
(48.00)\end{array}$ \\
\hline 3. & Three & $\begin{array}{c}39 \\
(39.00)\end{array}$ & $\begin{array}{c}31 \\
(31.00)\end{array}$ & $\begin{array}{c}70 \\
(35.00)\end{array}$ \\
\hline & Total & $\begin{array}{c}100 \\
(100.00)\end{array}$ & $\begin{array}{c}100 \\
(\mathbf{1 0 0 . 0 0})\end{array}$ & $\begin{array}{c}200 \\
(100.00)\end{array}$ \\
\hline
\end{tabular}

(Figures in parentheses indicate percentage to total) 
Table.7 Annual income of the family (Number of respondents)

\begin{tabular}{|c|c|c|c|c|}
\hline S.No & $\begin{array}{l}\text { Annual income } \\
\text { (Rs in lakhs) }\end{array}$ & Bangalore & Chennai & Overall \\
\hline 1. & $1.5-2.0$ & $\begin{array}{c}11 \\
(11.00)\end{array}$ & $\begin{array}{c}19 \\
(19.00)\end{array}$ & $\begin{array}{c}30 \\
(15.00)\end{array}$ \\
\hline 2. & 2.1-2.5 & $\begin{array}{c}27 \\
(27.00)\end{array}$ & $\begin{array}{c}26 \\
(26.00)\end{array}$ & $\begin{array}{c}65 \\
(32.50)\end{array}$ \\
\hline 3. & $2.6-3.0$ & $\begin{array}{c}39 \\
(39.00)\end{array}$ & $\begin{array}{c}32 \\
(32.00)\end{array}$ & $\begin{array}{c}59 \\
(29.50)\end{array}$ \\
\hline \multirow[t]{2}{*}{4.} & $3.1-3.5$ & $\begin{array}{c}23 \\
(23.00)\end{array}$ & $\begin{array}{c}23 \\
(23.00)\end{array}$ & $\begin{array}{c}46 \\
(23.00)\end{array}$ \\
\hline & Total & $\begin{array}{c}100 \\
(100.00)\end{array}$ & $\begin{array}{c}100 \\
(100.00)\end{array}$ & $\begin{array}{c}200 \\
(100.00)\end{array}$ \\
\hline
\end{tabular}

(Figures in parentheses indicate percentage to total)

Table.8 Purchase of brands of processed spice in Bangalore (Number of households)

\begin{tabular}{|c|c|c|c|c|c|c|}
\hline S.No & Brands & $\begin{array}{c}\text { Chilli } \\
\text { powder }\end{array}$ & $\begin{array}{l}\text { Dhaniya } \\
\text { powder }\end{array}$ & $\begin{array}{l}\text { Garam } \\
\text { masala }\end{array}$ & Paste & Others \\
\hline 1. & Brand-1 & $\begin{array}{c}19 \\
(19.00)\end{array}$ & $\begin{array}{c}13 \\
(13.00)\end{array}$ & $\begin{array}{c}0 \\
(0.00)\end{array}$ & $\begin{array}{c}0 \\
(0.00)\end{array}$ & $\begin{array}{c}43 \\
(43.00)\end{array}$ \\
\hline 2. & Brand-2 & $\begin{array}{c}81 \\
(81.00)\end{array}$ & $\begin{array}{c}87 \\
(87.00)\end{array}$ & $\begin{array}{c}18 \\
(18.00)\end{array}$ & $\begin{array}{c}0 \\
(0.00)\end{array}$ & $\begin{array}{c}13 \\
(13.00)\end{array}$ \\
\hline 3. & Brand-3 & $\begin{array}{c}0 \\
(0.00)\end{array}$ & $\begin{array}{c}0 \\
(0.00)\end{array}$ & $\begin{array}{c}72 \\
(72.00)\end{array}$ & $\begin{array}{c}0 \\
(0.00)\end{array}$ & $\begin{array}{c}0 \\
(0.00)\end{array}$ \\
\hline 4. & BRAND-4 & $\begin{array}{c}0 \\
(0.00)\end{array}$ & $\begin{array}{c}0 \\
(0.00)\end{array}$ & $\begin{array}{c}0 \\
(0.00)\end{array}$ & $\begin{array}{c}0 \\
(0.00)\end{array}$ & $\begin{array}{c}17 \\
(17.00)\end{array}$ \\
\hline 5. & Brand-5 & $\begin{array}{c}0 \\
(0.00)\end{array}$ & $\begin{array}{c}0 \\
(0.00)\end{array}$ & $\begin{array}{c}0 \\
(0.00)\end{array}$ & $\begin{array}{c}68 \\
(68.00)\end{array}$ & $\begin{array}{c}0 \\
(0.00)\end{array}$ \\
\hline 6. & Brand-6 & $\begin{array}{c}0 \\
(0.00)\end{array}$ & $\begin{array}{c}0 \\
(0.00)\end{array}$ & $\begin{array}{c}0 \\
(0.00)\end{array}$ & $\begin{array}{c}32 \\
(32.00)\end{array}$ & $\begin{array}{c}0 \\
(0.00)\end{array}$ \\
\hline \multirow[t]{2}{*}{7.} & Brand-7 & $\begin{array}{c}0 \\
(0.00)\end{array}$ & $\begin{array}{c}0 \\
(0.00)\end{array}$ & $\begin{array}{c}0 \\
(0.00)\end{array}$ & $\begin{array}{c}0 \\
(0.00)\end{array}$ & $\begin{array}{c}27 \\
(27.00)\end{array}$ \\
\hline & Total & $\begin{array}{c}100 \\
(100.00)\end{array}$ & $\begin{array}{c}100 \\
(100.00)\end{array}$ & $\begin{array}{c}100 \\
(100.00)\end{array}$ & $\begin{array}{c}100 \\
(100.00)\end{array}$ & $\begin{array}{c}100 \\
(100.00)\end{array}$ \\
\hline
\end{tabular}

(Figures in parentheses indicate percentage to total) 
Table.9 Purchase of brands of processed spice in Chennai (Number of households)

\begin{tabular}{|c|c|c|c|c|c|c|}
\hline S.No & Brands & $\begin{array}{c}\text { Chilli } \\
\text { powder }\end{array}$ & $\begin{array}{l}\text { Dhaniya } \\
\text { powder }\end{array}$ & $\begin{array}{l}\text { Garam } \\
\text { masala }\end{array}$ & Paste & Others \\
\hline 1. & Brand-1 & $\begin{array}{r}69 \\
(69.00)\end{array}$ & $\begin{array}{r}57 \\
(57.00)\end{array}$ & $\begin{array}{r}0 \\
(0.00)\end{array}$ & $\begin{array}{r}0 \\
(0.00)\end{array}$ & $\begin{array}{r}46 \\
(46.00)\end{array}$ \\
\hline 2. & BRAND-2 & $\begin{array}{r}13 \\
(13.00)\end{array}$ & $\begin{array}{r}19 \\
(19.00)\end{array}$ & $\begin{array}{r}17 \\
(17.00)\end{array}$ & $\begin{array}{r}0 \\
(0.00)\end{array}$ & $\begin{array}{r}23 \\
(23.00)\end{array}$ \\
\hline 3. & Brand-3 & $\begin{array}{r}0 \\
(0.00)\end{array}$ & $\begin{array}{r}0 \\
(0.00)\end{array}$ & $\begin{array}{r}49 \\
(49.00)\end{array}$ & $\begin{array}{r}0 \\
(0.00)\end{array}$ & $\begin{array}{r}0 \\
(0.00)\end{array}$ \\
\hline 4. & Brand-4 & $\begin{array}{r}0 \\
(0.00)\end{array}$ & $\begin{array}{r}0 \\
(0.00)\end{array}$ & $\begin{array}{r}0 \\
(0.00)\end{array}$ & $\begin{array}{r}0 \\
(0.00)\end{array}$ & $\begin{array}{r}21 \\
(21.00)\end{array}$ \\
\hline 5. & Brand-5 & $\begin{array}{r}0 \\
(0.00)\end{array}$ & $\begin{array}{r}0 \\
(0.00)\end{array}$ & $\begin{array}{r}0 \\
(0.00)\end{array}$ & $\begin{array}{r}69 \\
(69.00)\end{array}$ & $\begin{array}{r}0 \\
(0.00)\end{array}$ \\
\hline 6. & Brand-6 & $\begin{array}{r}0 \\
(0.00)\end{array}$ & $\begin{array}{r}0 \\
(0.00)\end{array}$ & $\begin{array}{r}0 \\
(0.00)\end{array}$ & $\begin{array}{r}31 \\
(31.00)\end{array}$ & $\begin{array}{r}0 \\
(0.00)\end{array}$ \\
\hline 7. & Brand-7 & $\begin{array}{r}18 \\
(18.00)\end{array}$ & $\begin{array}{r}15 \\
(15.00)\end{array}$ & $\begin{array}{r}23 \\
(23.00)\end{array}$ & $\begin{array}{r}0 \\
(0.00)\end{array}$ & $\begin{array}{r}10 \\
(10.000\end{array}$ \\
\hline 8. & Brand-8 & $\begin{array}{r}0 \\
(0.00)\end{array}$ & $\begin{array}{r}9 \\
(9.00)\end{array}$ & $\begin{array}{r}0 \\
(0.00)\end{array}$ & $\begin{array}{r}0 \\
(0.00)\end{array}$ & $\begin{array}{r}0 \\
(0.00)\end{array}$ \\
\hline 9. & Brand-9 & $\begin{array}{r}0 \\
(0.00)\end{array}$ & $\begin{array}{r}0 \\
(0.00)\end{array}$ & $\begin{array}{r}11 \\
(11.00)\end{array}$ & $\begin{array}{r}0 \\
(0.00)\end{array}$ & $\begin{array}{r}0 \\
(0.00)\end{array}$ \\
\hline & Total & $\begin{array}{r}100 \\
(100.00)\end{array}$ & $\begin{array}{r}100 \\
(100.00)\end{array}$ & $\begin{array}{r}100 \\
(100.00)\end{array}$ & $\begin{array}{r}100 \\
(100.00)\end{array}$ & $\begin{array}{r}100 \\
(100.00)\end{array}$ \\
\hline
\end{tabular}

(Figures in parentheses indicate percentage to total)

Table.10 Consumption of different processed spice products in Bangalore and Chennai

\begin{tabular}{|c|c|c|c|c|c|c|}
\hline \multirow[t]{2}{*}{ S.No } & \multirow[t]{2}{*}{ Brands } & \multirow{2}{*}{$\begin{array}{c}\text { Processed } \\
\text { products }\end{array}$} & \multicolumn{2}{|c|}{ Bangalore } & \multicolumn{2}{|c|}{ Chennai } \\
\hline & & & $\begin{array}{l}\text { Consumption } \\
\text { (in kgs) }\end{array}$ & Rank & $\begin{array}{l}\text { Consumption } \\
\text { (in kgs) }\end{array}$ & Rank \\
\hline 1. & Brand-7 & Other powders & 8.45 & 1 & 10.08 & 5 \\
\hline 2. & Brand-7 & Dhaniya powder & 9.20 & 2 & 10.23 & 6 \\
\hline 3. & Brand-7 & Chilli powder & 11.31 & 3 & 9.85 & 4 \\
\hline 4. & Brand-6 & Ginger paste & 12.45 & 4 & 11.54 & 7 \\
\hline 5. & Brand-5 & Ginger paste & 17.50 & 5 & 16.85 & 10 \\
\hline 6. & Brand-1 & Chilli powder & 18.80 & 6 & 21.87 & 11 \\
\hline 7. & Brand-1 & Dhaniya powder & 21.25 & 7 & 27.65 & 13 \\
\hline 8. & Brand-1 & Other powders & 24.71 & 8 & 39.85 & 15 \\
\hline 9. & BRAND-2 & Dhaniya powder & 29.17 & 9 & 14.51 & 9 \\
\hline 10. & BRAND-2 & Chilli powder & 35.25 & 10 & 12.34 & 8 \\
\hline 11. & Brand-3 & Gram masala & 38.75 & 11 & 38.34 & 14 \\
\hline 12. & Brand-9 & Chilli powder & - & - & 7.35 & 2 \\
\hline 13. & Brand-9 & Dhaniya powder & - & - & 8.67 & 3 \\
\hline 14. & Brand-9 & Other powders & - & - & 7.15 & 1 \\
\hline
\end{tabular}

$(\mu=90)$

$\left(\mathrm{n}_{1}=12 ; \sum \mathrm{R}_{1}=78\right.$; calculated $\left.\mathrm{U}_{1}=180 ; \mathrm{Z}_{1}=0.192\right)$

$\left(\mathrm{n}_{2}=15 ; \sum \mathrm{R}_{2}=105 ;\right.$ calculated $\left.\mathrm{U}_{2}=195 ; \mathrm{Z}_{2}=1.217\right)$ 
Table.11 Reasons for buying preferred brands in Bangalore (Number of households)

\begin{tabular}{|c|c|c|c|c|c|c|c|c|}
\hline S.No & Reasons & Brand-1 & Brand-2 & Brand-3 & Brand-4 & Brand-5 & Brand-6 & Brand-7 \\
\hline 1. & $\begin{array}{l}\text { Brand } \\
\text { loyalty }\end{array}$ & $\begin{array}{c}23 \\
(30.66)\end{array}$ & $\begin{array}{c}31 \\
(35.63)\end{array}$ & $\begin{array}{c}16 \\
(22.22)\end{array}$ & $\begin{array}{c}9 \\
(52.94)\end{array}$ & $\begin{array}{c}37 \\
(71.15)\end{array}$ & $\begin{array}{c}12 \\
(57.14)\end{array}$ & $\begin{array}{c}6 \\
(22.22)\end{array}$ \\
\hline 2. & $\begin{array}{l}\text { Good } \\
\text { quality }\end{array}$ & $\begin{array}{c}49 \\
(65.33)\end{array}$ & $\begin{array}{c}58 \\
(66.67)\end{array}$ & $\begin{array}{c}43 \\
(59.72)\end{array}$ & $\begin{array}{c}12 \\
(70.58)\end{array}$ & $\begin{array}{c}32 \\
(61.54)\end{array}$ & $\begin{array}{c}18 \\
(85.71)\end{array}$ & $\begin{array}{c}11 \\
(40.74)\end{array}$ \\
\hline 3. & Flavour & $\begin{array}{c}11 \\
(14.66)\end{array}$ & $\begin{array}{c}39 \\
(44.83)\end{array}$ & $\begin{array}{c}17 \\
(23.61)\end{array}$ & $\begin{array}{c}10 \\
(58.82)\end{array}$ & $\begin{array}{c}21 \\
(40.34)\end{array}$ & $\begin{array}{c}16 \\
(76.19)\end{array}$ & $\begin{array}{c}8 \\
(29.62)\end{array}$ \\
\hline 4. & Taste & $\begin{array}{c}31 \\
(41.33)\end{array}$ & $\begin{array}{c}32 \\
(36.78)\end{array}$ & $\begin{array}{c}25 \\
(34.72)\end{array}$ & $\begin{array}{c}11 \\
(64.70)\end{array}$ & $\begin{array}{c}26 \\
(50.00)\end{array}$ & $\begin{array}{c}13 \\
(61.90)\end{array}$ & $\begin{array}{c}7 \\
(25.92)\end{array}$ \\
\hline 5. & $\begin{array}{l}\text { Product } \\
\text { variety }\end{array}$ & $\begin{array}{c}28 \\
(37.33)\end{array}$ & $\begin{array}{c}23 \\
(26.44)\end{array}$ & $\begin{array}{c}31 \\
(43.05)\end{array}$ & $\begin{array}{c}6 \\
(35.29)\end{array}$ & $\begin{array}{c}5 \\
(9.61)\end{array}$ & $\begin{array}{c}6 \\
(28.57)\end{array}$ & $\begin{array}{c}10 \\
(37.03)\end{array}$ \\
\hline 6. & Pack size & $\begin{array}{c}9 \\
(12.00)\end{array}$ & $\begin{array}{c}11 \\
(12.64)\end{array}$ & $\begin{array}{c}10 \\
(13.88)\end{array}$ & $\begin{array}{c}0 \\
(0.00)\end{array}$ & $\begin{array}{c}8 \\
(15.38)\end{array}$ & $\begin{array}{c}7 \\
(33.34)\end{array}$ & $\begin{array}{c}5 \\
(18.51)\end{array}$ \\
\hline 7. & $\begin{array}{l}\text { Brand } \\
\text { image }\end{array}$ & $\begin{array}{c}36 \\
(48.00)\end{array}$ & $\begin{array}{c}44 \\
(50.57)\end{array}$ & $\begin{array}{c}12 \\
(16.66)\end{array}$ & $\begin{array}{c}9 \\
(52.94)\end{array}$ & $\begin{array}{c}29 \\
(55.76)\end{array}$ & $\begin{array}{c}11 \\
(52.38)\end{array}$ & $\begin{array}{c}9 \\
(33.33)\end{array}$ \\
\hline 8. & $\begin{array}{l}\text { Product } \\
\text { features }\end{array}$ & $\begin{array}{c}17 \\
(22.66)\end{array}$ & $\begin{array}{c}19 \\
(21.84)\end{array}$ & $\begin{array}{c}9 \\
(12.50)\end{array}$ & $\begin{array}{c}5 \\
(29.41)\end{array}$ & $\begin{array}{c}11 \\
(21.15)\end{array}$ & $\begin{array}{c}9 \\
(42.85)\end{array}$ & $\begin{array}{c}5 \\
(18.51)\end{array}$ \\
\hline 9. & Shelf life & $\begin{array}{c}15 \\
(20.00)\end{array}$ & $\begin{array}{c}31 \\
(35.63)\end{array}$ & $\begin{array}{c}19 \\
(26.38)\end{array}$ & $\begin{array}{c}8 \\
(47.05)\end{array}$ & $\begin{array}{c}12 \\
(23.07)\end{array}$ & $\begin{array}{c}12 \\
(57.14)\end{array}$ & $\begin{array}{c}11 \\
(40.74)\end{array}$ \\
\hline 10. & $\begin{array}{l}\text { Attractive } \\
\text { packs }\end{array}$ & $\begin{array}{c}33 \\
(44.00)\end{array}$ & $\begin{array}{c}48 \\
(55.17)\end{array}$ & $\begin{array}{c}26 \\
(36.11)\end{array}$ & $\begin{array}{c}7 \\
(41.17)\end{array}$ & $\begin{array}{c}19 \\
(36.53)\end{array}$ & $\begin{array}{c}10 \\
(47.61)\end{array}$ & $\begin{array}{c}15 \\
(55.55)\end{array}$ \\
\hline 11. & Price & $\begin{array}{c}18 \\
(24.00)\end{array}$ & $\begin{array}{c}19 \\
(21.84)\end{array}$ & $\begin{array}{c}12 \\
(16.66)\end{array}$ & $\begin{array}{c}0 \\
(0.00)\end{array}$ & $\begin{array}{c}12 \\
(23.07)\end{array}$ & $\begin{array}{c}7 \\
(33.34)\end{array}$ & $\begin{array}{c}9 \\
(\mathbf{3 3 . 3 3})\end{array}$ \\
\hline & $\mathbf{n}$ & $\begin{array}{c}75 \\
(100.00)\end{array}$ & $\begin{array}{c}87 \\
(100.00)\end{array}$ & $\begin{array}{c}72 \\
(100.00)\end{array}$ & $\begin{array}{c}17 \\
(100.00)\end{array}$ & $\begin{array}{c}52 \\
(100.00)\end{array}$ & $\begin{array}{c}21 \\
(100.00)\end{array}$ & $\begin{array}{c}27 \\
(100.00)\end{array}$ \\
\hline
\end{tabular}

(Figures in parentheses indicate percentage to number of users of the respective brand (n) in each city) 
Table.12 Reasons for buying above brands in Chennai (Number of households)

\begin{tabular}{|c|c|c|c|c|c|c|c|c|c|c|}
\hline S.No & Reasons & Brand-1 & Brand-2 & Brand-3 & Brand-4 & Brand-5 & Brand-6 & Brand-7 & Brand-3 & Brand-9 \\
\hline 1. & Brand loyalty & $\begin{array}{c}73 \\
(82.02)\end{array}$ & $\begin{array}{c}27 \\
(37.50)\end{array}$ & $\begin{array}{c}32 \\
(65.30)\end{array}$ & $\begin{array}{c}8 \\
(38.10)\end{array}$ & $\begin{array}{c}28 \\
(57.14)\end{array}$ & $\begin{array}{c}10 \\
(47.62)\end{array}$ & $\begin{array}{c}17 \\
(47.22)\end{array}$ & $\begin{array}{c}0 \\
(0.00)\end{array}$ & $\begin{array}{c}0 \\
(\mathbf{0 . 0 0})\end{array}$ \\
\hline 2. & Good quality & $\begin{array}{c}67 \\
(75.28)\end{array}$ & $\begin{array}{c}38 \\
(52.78)\end{array}$ & $\begin{array}{c}41 \\
(83.67)\end{array}$ & $\begin{array}{c}11 \\
(52.38)\end{array}$ & $\begin{array}{c}43 \\
(87.75)\end{array}$ & $\begin{array}{c}15 \\
(77.43)\end{array}$ & $\begin{array}{c}24 \\
(66.67)\end{array}$ & $\begin{array}{c}7 \\
(77.77)\end{array}$ & $\begin{array}{c}9 \\
(81.81)\end{array}$ \\
\hline 3. & Flavour & $\begin{array}{c}28 \\
(31.46)\end{array}$ & $\begin{array}{c}25 \\
(34.72)\end{array}$ & $\begin{array}{c}35 \\
(71.43)\end{array}$ & $\begin{array}{c}9 \\
(42.86)\end{array}$ & $\begin{array}{c}39 \\
(79.59)\end{array}$ & $\begin{array}{c}12 \\
(57.15)\end{array}$ & $\begin{array}{c}22 \\
(61.11)\end{array}$ & $\begin{array}{c}0 \\
(0.00)\end{array}$ & $\begin{array}{c}5 \\
(45.45)\end{array}$ \\
\hline 4. & Taste & $\begin{array}{c}39 \\
(43.82)\end{array}$ & $\begin{array}{c}31 \\
(43.06)\end{array}$ & $\begin{array}{c}28 \\
(57.14)\end{array}$ & $\begin{array}{c}7 \\
(33.33)\end{array}$ & $\begin{array}{c}35 \\
(71.42)\end{array}$ & $\begin{array}{c}13 \\
(61.90)\end{array}$ & $\begin{array}{c}29 \\
(80.55)\end{array}$ & $\begin{array}{c}5 \\
(55.55)\end{array}$ & $\begin{array}{c}6 \\
(54.54)\end{array}$ \\
\hline 5. & $\begin{array}{l}\text { Product } \\
\text { variety }\end{array}$ & $\begin{array}{c}81 \\
(91.01)\end{array}$ & $\begin{array}{c}22 \\
(30.56)\end{array}$ & $\begin{array}{c}36 \\
(73.47)\end{array}$ & $\begin{array}{c}6 \\
(28.57)\end{array}$ & $\begin{array}{c}19 \\
(38.77)\end{array}$ & $\begin{array}{c}7 \\
(33.32)\end{array}$ & $\begin{array}{c}31 \\
(86.11)\end{array}$ & $\begin{array}{c}0 \\
(0.00)\end{array}$ & $\begin{array}{c}0 \\
(0.00)\end{array}$ \\
\hline 6. & Pack size & $\begin{array}{c}13 \\
(14.61)\end{array}$ & $\begin{array}{c}17 \\
(23.61)\end{array}$ & $\begin{array}{c}11 \\
(22.45)\end{array}$ & $\begin{array}{c}0 \\
(0.00)\end{array}$ & $\begin{array}{c}12 \\
(24.48)\end{array}$ & $\begin{array}{c}0 \\
(0.00)\end{array}$ & $\begin{array}{c}14 \\
(38.89)\end{array}$ & $\begin{array}{c}0 \\
(0.00)\end{array}$ & $\begin{array}{c}0 \\
(0.00)\end{array}$ \\
\hline 7. & Brand image & $\begin{array}{c}41 \\
(48.31)\end{array}$ & $\begin{array}{c}32 \\
(44.44)\end{array}$ & $\begin{array}{c}24 \\
(48.98)\end{array}$ & $\begin{array}{c}0 \\
(0.00)\end{array}$ & $\begin{array}{c}32 \\
(65.30)\end{array}$ & $\begin{array}{c}14 \\
(66.67)\end{array}$ & $\begin{array}{c}15 \\
(41.67)\end{array}$ & $\begin{array}{c}0 \\
(0.00)\end{array}$ & $\begin{array}{c}0 \\
(0.00)\end{array}$ \\
\hline 8. & $\begin{array}{l}\text { Product } \\
\text { features }\end{array}$ & $\begin{array}{c}38 \\
(42.70)\end{array}$ & $\begin{array}{c}19 \\
(26.39)\end{array}$ & $\begin{array}{c}16 \\
(32.65)\end{array}$ & $\begin{array}{c}0 \\
(0.00)\end{array}$ & $\begin{array}{c}11 \\
(22.45)\end{array}$ & $\begin{array}{c}9 \\
(42.86)\end{array}$ & $\begin{array}{c}10 \\
(27.78)\end{array}$ & $\begin{array}{c}0 \\
(0.00)\end{array}$ & $\begin{array}{c}0 \\
(\mathbf{0 . 0 0})\end{array}$ \\
\hline 9. & Shelf life & $\begin{array}{c}35 \\
(39.32)\end{array}$ & $\begin{array}{c}21 \\
(29.16)\end{array}$ & $\begin{array}{c}18 \\
(36.73)\end{array}$ & $\begin{array}{c}10 \\
(47.62)\end{array}$ & $\begin{array}{c}24 \\
(48.98)\end{array}$ & $\begin{array}{c}12 \\
(57.15)\end{array}$ & $\begin{array}{c}18 \\
(50.00)\end{array}$ & $\begin{array}{c}8 \\
(88.89)\end{array}$ & $\begin{array}{c}5 \\
(45.45)\end{array}$ \\
\hline 10. & $\begin{array}{l}\text { Attractive } \\
\text { packs }\end{array}$ & $\begin{array}{c}26 \\
(29.21)\end{array}$ & $\begin{array}{c}17 \\
(23.61)\end{array}$ & $\begin{array}{c}20 \\
(40.82)\end{array}$ & $\begin{array}{c}6 \\
(28.57)\end{array}$ & $\begin{array}{c}19 \\
(38.78)\end{array}$ & $\begin{array}{c}8 \\
(38.10)\end{array}$ & $\begin{array}{c}22 \\
(61.11)\end{array}$ & $\begin{array}{c}0 \\
(0.00)\end{array}$ & $\begin{array}{c}0 \\
(\mathbf{0 . 0 0})\end{array}$ \\
\hline \multirow[t]{2}{*}{11.} & Price & $\begin{array}{c}30 \\
(33.70)\end{array}$ & $\begin{array}{c}19 \\
(26.39)\end{array}$ & $\begin{array}{c}15 \\
(30.61)\end{array}$ & $\begin{array}{c}0 \\
(0.00)\end{array}$ & $\begin{array}{c}9 \\
(18.37)\end{array}$ & $\begin{array}{c}0 \\
(0.00)\end{array}$ & $\begin{array}{c}12 \\
(33.33)\end{array}$ & $\begin{array}{c}0 \\
(0.00)\end{array}$ & $\begin{array}{c}0 \\
(0.00)\end{array}$ \\
\hline & $\mathbf{n}$ & $\begin{array}{c}89 \\
(100.0)\end{array}$ & $\begin{array}{c}72 \\
(100.0)\end{array}$ & $\begin{array}{c}49 \\
(100.0)\end{array}$ & $\begin{array}{c}21 \\
(100.0)\end{array}$ & $\begin{array}{c}49 \\
(100.0)\end{array}$ & $\begin{array}{c}21 \\
(100.0)\end{array}$ & $\begin{array}{c}36 \\
(100.0)\end{array}$ & $\begin{array}{c}9 \\
(100.0)\end{array}$ & $\begin{array}{c}11 \\
(100.0)\end{array}$ \\
\hline
\end{tabular}

(Figures in parentheses indicate percentage to number of users of the respective brand (n) in each city) 


\section{Purchase of processed spice brands}

The processed spice products include Chilli powder, Dhaniya powder, Garam masala, pastes such as ginger paste, ginger garlic paste, tamarind paste etc., and other powders like chicken masala, mutton masala and other non-vegetarian items were grouped under ' other powders'. Different brands of spice products purchased and used for culinary purposes by respondents was analysed. All the respondents use one or more processed spice products for cooking. Details about purchase of brands of processed spices at Bangalore and Chennai are presented in Tables 8 and 9 .

Of the total sample respondents more than 80 per cent purchased Brand-2 Chilli and Dhaniya powders followed by Brand-1 in Bangalore. Brand-3 was the ruling brand in garam masala whereas most of the people preferred Brand-5 ginger paste for cooking purpose. Brand-1 ruled most of the nonvegetarian recepies.

In contrast to Bangalore, Brand-1 was ruling as number one in Chennai. It could be noted that Brand-1 Chilli and Dhaniya powders was predominantly used by the sample respondents followed by the emerging brand Brand-7 and BRAND-2. About 50 per cent of the households used Brand-3 gram masala whereas the rest of the households used Brand7 (23 per cent), Brand-2 (17 per cent) and Brand-9 (11 per cent). In Chennai market also Brand-5 had first position for its ginger paste. Brand-4 (21.00 per cent) was competing with Brand-2 (23.00 per cent) in the others category.

\section{Mann Whitney test}

Mann Whitney test was used to test whether there was significant difference between the consumption (per month) of processed spice products between sample respondents of Bangalore and Chennai. The consumption of different brands were ranked and analysed. The results are presented in Table 10. The consumption particulars relate to the total consumption by 100 households in Chennai and Bangalore each.

As the observed value of $U_{1}$ and $U_{2}$ was greater than the table value, the null hypothesis was rejected. It could be concluded that the brands of processed spices consumed in two cities were significantly different.

\section{Reasons for buying preferred brands}

Different people in both the cities preferred different brands of processed spice products. The respondents who used the brands were asked to state the reasons for buying those brands. The data was analyzed and the results for Bangalore and Chennai are presented in Tables 11 and 12.

It could be concluded that (Table 11) BRAND-2, Brand-1, Brand-3 were the major brands purchased in Bangalore. Good quality (66.67 per cent) followed by attractive packs (55.17 per cent), brand image (50.57 per cent) were the main reasons for buying BRAND-2 brands. Brand-1 products were bought for its good quality (65.33 per cent), brand image (48.00 per cent) and attractive packs (55.17 per cent). Brand-3 brands were preferred mainly for its good quality (59.72 per cent), product variety (43.05 per cent) and attractive packs (36.11 per cent). Overall the above brands were brought for its good quality, brand image and attractive package.

\section{Reasons for buying preferred brands in Chennai}

Majority of the sample respondents in Chennai purchased Brand-1 for its product 
variety (91.01 per cent) followed by brand loyalty (82.02 per cent) and also for its good quality (75.28 per cemt). BRAND-2 brand was bought by most of the respondents due to its quality (52.78 per cent), brand image (44.44 per cent) and taste (43.06 per cent). Brand-3 was preferred mostly for its good quality (83.67 per cent) followed by product variety (73.47 per cent) and flavour (71.43 per cent). The most common reasons for the purchase of major brands were good quality and product variety.

Considering the reasons for selection of brands at Bangalore and Chennai it could be concluded that good quality, product variety, brand image and attractive package are essential to attract customers.

In conclusion the majority of the sample respondents belonged to the age group of 3655 years. About 50 per cent of the respondents were graduates. Around 57 per cent of total respondents were employed. Majority of the respondents (91 per cent) belonged to the nuclear family category. The family income of about 33 per cent of the total respondents was Rs 2.1 to 2.5 lakhs per annum. Considering the reasons for selection of brands at Bangalore and Chennai it could be concluded that good quality, product variety, brand image and attractive package are essential to attract customers. Brands of processed spices consumed in two cities were significantly different.

\section{References}

Spinelli, S., Masi, C., Zoboli, G. P., Prescott, J., and Monteleone, E. (2015). Emotional responses to branded and unbranded foods. Food Quality and Preference, 42, 1-11.

Xie, B., Wang, L., Yang, H., Wang, Y., \& Zhang, M. (2015). Consumer perceptions and attitudes of organic food products in Eastern China. British Food Journal, 117(3), 1105-1121.

ST Wang, E. (2013). The influence of visual packaging design on perceived food product quality, value, and brand preference. International Journal of Retail \& Distribution Management, 41(10), 805-816.

Liu, Richie L.; Sprott, David E.; Spangenberg, Eric R.; Czellar, Sandor; and Voss, Kevin E., 2018. "Consumer preference for national vs. private brands: The influence of brand engagement and self-concept threat," Journal of Retailing and Consumer Services, Elsevier, vol. 41(C), pages 90-100.

Mohan Kathuria, L., and Gill, P. (2013). Purchase of branded commodity food products: empirical evidence from India. British Food Journal, 115(9), 1255-1280.

\section{How to cite this article:}

Indumathi, V. M., M. Malarkodi and Jesupriya Poornakala, S. 2020. A Comparative Study on Consumers' Preference of Processed Spices in Bangalore and Chennai City. Int.J.Curr.Microbiol.App.Sci. 9(11): 2418-2429. doi: https://doi.org/10.20546/ijcmas.2020.911.291 